\title{
Determining the feasibility of a trial to evaluate the effectiveness of phototherapy versus placebo at reducing pain during physical activity for people with knee osteoarthritis: a pilot randomized controlled trial
}

Kyle Vader ${ }^{1,2}$, Abey Bekele Abebe ${ }^{1}$, Mulugeta Bayisa Chala', Kevin Varette ${ }^{1}$ and Jordan Miller ${ }^{1 *}$

\begin{abstract}
Background: Although practice guidelines recommend physical activity and exercise for the management of knee osteoarthritis, pain is a common barrier to participation. Phototherapy has been shown to reduce pain intensity for people with knee osteoarthritis, but it is unclear if it reduces pain during physical activity or contributes to improved rehabilitation outcomes.

Objective: The aim of this study is to assess the feasibility of performing a fully powered randomized controlled trial $(R C T)$ comparing an active phototherapy intervention versus placebo on pain during physical activity for people with knee osteoarthritis.

Methods: A pilot RCT was conducted to test the feasibility of a trial comparing 8-sessions (4 weeks) of active phototherapy versus placebo. People were able to participate if they (1) were an English speaking adult (> 18 years of age), (2) had received a diagnosis of knee osteoarthritis from a physician, and (3) self-reported experiencing pain and disability related to their knee osteoarthritis for $>3$ months. Primary outcomes were the feasibility of participant recruitment, retention, assessment procedures, and maintaining high treatment fidelity. Secondary outcomes piloted for a full trial included pain during physical activity (primary outcome of full trial); self-reported pain severity, physical function, stiffness, adherence to prescribed exercise, global rating of change, patient satisfaction, and adverse events; 6-min walk test; and pressure pain threshold.
\end{abstract}

*Correspondence: jordan.miller@queensu.ca

'School of Rehabilitation Therapy, Queen's University, Kingston, ON, Canada

Full list of author information is available at the end of the article

C C The Author(s). 2020 Open Access This article is licensed under a Creative Commons Attribution 4.0 International License, which permits use, sharing, adaptation, distribution and reproduction in any medium or format, as long as you give appropriate credit to the original author(s) and the source, provide a link to the Creative Commons licence, and indicate if changes were made. The images or other third party material in this article are included in the article's Creative Commons licence, unless indicated otherwise in a credit line to the material. If material is not included in the article's Creative Commons licence and your intended use is not permitted by statutory regulation or exceeds the permitted use, you will need to obtain permission directly from the copyright holder. To view a copy of this licence, visit http://creativecommons.org/licenses/by/4.0/. The Creative Commons Public Domain Dedication waiver (http://creativecommons.org/publicdomain/zero/1.0/) applies to the data made available in this article, unless otherwise stated in a credit line to the data. 
(Continued from previous page)

Results: Twenty participants (4 men; 16 women) with knee osteoarthritis and a mean age of 63.95 (SD: 9.27) years were recruited over a 3-week period (6.7 participants per week). Fifteen out of 20 (75\%) of participants completed the primary outcome assessment at 4 weeks and 19/20 (95\%) of participants were retained and completed the final 16-week assessment. Overall, 89\% of all assessment items were completed by participants across all time-points. Fifteen out of 20 participants (75\%) completed all 8 treatment sessions. Treatment fidelity was 100\% for all completed treatment sessions. No adverse events were reported by participants in either group.

Conclusions: Results suggest that the trial methodology and intervention are feasible for implementation in a fully powered randomized controlled trial to determine the effectiveness of phototherapy at reducing pain during physical activity for people with knee osteoarthritis.

Trial registration: ClinicalTrials.gov, NCT04234685, January 21, 2020-Retrospectively registered.

Keywords: Phototherapy, Knee osteoarthritis, Sensitivity to physical activity, Pain, disability

\section{Key messages regarding feasibility}

- Prior to this research, the feasibility of performing a fully powered randomized controlled trial comparing an active phototherapy intervention versus placebo to determine the effectiveness of phototherapy at reducing pain during physical activity for people with knee osteoarthritis was unknown.

- Results suggest the trial methodology and intervention are feasible for implementation in a fully powered randomized controlled trial to determine the effectiveness of phototherapy at reducing pain during physical activity for people with knee osteoarthritis.

- Results have the following implications for a fully powered randomized controlled trial: (1) we will aim to recruit participants from primary care sites where they have the physical space available to complete the 6-min walk test onsite when necessary to overcome geographic barriers to in-person assessments in order to achieve higher rates of completion for the primary outcome; (2) we will follow-up multiple times via email and telephone with participants who are unable to attend in-person assessments to ensure that we still achieve high rates of completion of selfreport measures; and (3) we will update our sample size calculation in order to allow for up to $25 \%$ dropout from in-person sessions and/or assessments.

\section{Introduction}

Musculoskeletal disorders are the leading contributor to years lived with disability worldwide [1-3]. Musculoskeletal disorders also have a significant economic burden on health care systems [4] and an even greater impact on society due to time off work [5-8]. As the number of older adults grows, the burden of osteoarthritis $(\mathrm{OA})$ is only projected to rise [4].

Clinical practice guidelines recommend aerobic and strengthening exercise for people with knee OA [9-15]. Exercise is an effective non-surgical intervention for OA that can decrease pain and improve physical function [9-15]. However, adherence to physical activity and exercise interventions are low in this population [16-19]. Pain during physical activity is a frequently cited barrier that can interfere with adherence [20-22]. Effective treatment options to reduce pain during physical activity may help overcome barriers to participation in physical activity and exercise among this population.

Evidence suggests that pain with movement or physical activity may be a distinct construct from pain at rest [23] including differing mechanisms and pain processing [24-26]. There is currently a dearth of evidence on the effect of clinical interventions on reducing pain during physical activity; however, some early evidence suggests that pain during physical activity may respond differently to interventions than pain at rest. For example, evidence suggests that opioids, commonly used in the treatment of post-surgical pain, have a very small impact on pain during movement for people with postsurgical pain [27]. On the other hand, transcutaneous electrical nerve stimulation (TENS) [28] and acetaminophen [29] have been demonstrated to be potential modulators of pain during movement. Pain during physical activity is often not assessed as a distinct construct in clinical trials on OA, but is an important barrier to participation in physical activity and exercise interventions that requires further study.

Phototherapy may help to address the need for effective, non-pharmacological, pain-relieving interventions in people with knee OA [30-32]. Although the physiological mechanisms of pain relief are not clear, phototherapy has been shown to reduce inflammation, induce analgesia, and promote healing of musculoskeletal disorders $[33,34]$. One hypothesis is that phototherapy can increase nociceptive thresholds, which can result in subsequent pain relief [35]. Phototherapy has demonstrated improvements in pain for several musculoskeletal disorders such as low back and neck pain [36-40], suggesting it may be a valuable component in the management of knee OA. However, research on the effectiveness of phototherapy on pain during physical activity remains 
unknown. Before proceeding with a fully powered randomized controlled trial to evaluate the effectiveness of phototherapy on pain during physical activity, we set out to determine the feasibility of conducting such a trial.

Primary outcomes of this pilot study were feasibility measures including participant recruitment rate, assessment completion rate, retention rate, and treatment fidelity. Secondary outcomes piloted for a full trial included pain during physical activity (primary outcome for the full trial), pain severity, pressure pain threshold, functional walking performance, and self-reported physical function stiffness, pain, adherence to prescribed exercise, global rating of change, patient satisfaction, and adverse events.

\section{Methods}

\section{Design}

The study was designed as a parallel group, pilot randomized controlled trial with 1:1 allocation ratio to active phototherapy intervention or placebo.

\section{Recruitment}

Participants were recruited from two primary care practices in Kingston, Ontario, Canada. People who called the primary care clinics were asked for their "reason for visit" (as is standard practice). When the reason for visit was knee OA, people were asked by their physician or nurse if they were willing to be contacted by a research assistant to be invited to participate in this research. If they agreed to being invited to participate, they were asked to provide contact information for a research assistant to contact them. The research assistant then called them, screened them for eligibility over the telephone, and invited eligible participants to an initial visit where agreeable participants provided informed consent and completed an initial assessment.

\section{Sample size}

Our targeted sample size was 20 participants. As this was a pilot study, a formal sample size calculation was not conducted. A sample of 20 participants was chosen because researchers and clinical partners felt this would be a large enough sample to allow an accurate assessment of the feasibility of recruitment, retention, outcome measure completion, and delivering the phototherapy and exercise interventions. Additionally, this sample size meets the recommendation from Whitehead et al. of a minimum of 10 participants per arm when estimating the variance for a sample size calculation for a future trial designed with $90 \%$ power, two-sided $5 \%$ significance, and a large (0.8) effect size [41]. Estimating the variance was not a primary objective of this study and given the uncertainty of the variance estimated from such a small sample, variance estimates from previous research on interventions to improve pain and function for people with knee OA will also be used to inform our sample size for the future trial.

\section{Inclusion criteria}

English speaking adults ( $>18$ years of age) who had been given a diagnosis of knee OA by a physician and had experienced pain and disability related to their knee OA for at least 3 months in duration were able to participate in this research.

\section{Exclusion criteria}

Participants were excluded if they had a skin condition or other condition for which the application of phototherapy may have been contra-indicated (e.g., skin cancer or people receiving radiation therapy), history of total joint arthroplasty in the affected knee, and "red flags" suggestive of non-musculoskeletal etiology (i.e., fever/chills, unrelenting night pain, multi-segmental or bilateral loss of sensation, or sudden and unexplained weight loss).

\section{Research ethics}

This study was approved by the Queen's University Health Sciences and Affiliated Teaching Hospitals Research Ethics Board in Kingston, Ontario, Canada (Reference Number: 6022509). All participants provided written informed consent prior to participation in this research.

\section{Randomization and allocation concealment}

Participants were randomized using a computer-generated block random number generator with random block sizes by a study team member not involved in the assessments, allocation, or interventions. The allocation sequence was recorded on cards and placed in sequentially numbered opaque envelopes that were sealed. Allocation was concealed until after the initial assessment was completed. A research assistant opened the sealed envelope that said either "group A" or "group B" after the initial assessment without knowledge of whether "group A" or "group B" would receive the active or placebo phototherapy.

\section{Blinding}

Participants and research assistants were blinded to whether participants received the active phototherapy intervention or placebo. The phototherapy machine was pre-programmed with two dosage settings for "group A" or "group B." Both settings involved emission of a visible red light. The phototherapy started after a programmed 15-s delay to allow the research assistant to exit the room prior to the treatment starting. This prevented the research assistant from seeing the difference in light intensity between intervention and control groups. Whether the "group A" or "group B" was the active phototherapy or placebo dosage was not revealed to the research assistant or participant at any point during the research study. 


\section{Intervention}

\section{Exercise and education}

Participants from both the active phototherapy intervention and placebo groups took part in a standardized education and exercise intervention as suggested by best evidence and clinical practice guidelines for knee OA $[12,15]$. These sessions were approximately $20 \mathrm{~min}$ in length and took place twice weekly for 4 weeks (8 sessions) with a trained physiotherapist.

Education Participants received education on evidencebased management of knee OA at the first visit [12, 15]. This education included describing the importance of regular physical activity in managing knee OA and how our bodies can adapt to gradual increases in activity and exercise. It also included education on how temporary increases in pain or soreness after activity can be normal when starting an exercise program. The education was provided at the first treatment session and took approximately $10 \mathrm{~min}$.

Aerobic exercise Participants were provided with a tailored home walking program [10, 42]. Participants were given instructions at their initial visit and these instructions were re-inforced at each subsequent visit. For those who were able, participants were instructed to walk 30 min 5 times per week in order to meet physical activity guideline recommendations [43, 44]. All participants who reported being unable to achieve this walking duration (e.g., pain or physical disability interfered with the ability to walk for $30 \mathrm{~min}$ ) were provided with a progressive walking program [45]. The progressive walking program started at their baseline, defined as the distance they felt confident they could walk without being sore an hour later and without feeling like they would be unable to perform their usual activities of daily living during the remainder of the day because of the walk. Participants worked with the physiotherapist to develop a progressive walking program that progressed by $10-20 \%$ each week from this baseline until they reached 30 min per day.

\section{Strengthening, balance, and range of motion} exercises Participants were instructed how to perform a series of six land-based exercises that were consistent with clinical practice guidelines for knee OA [11, 12, 15], including strengthening (squat to chair, step-up, lunge, resisted knee flexion), balance (single-leg stance), and range of motion (terminal knee flexion/extension range of motion) exercises. The exercise technique and dosage were tailored to the participant's current abilities under the supervision of a physiotherapist. For example, if a participant was unable to squat down to the level of a chair and return to standing, the chair height was raised until the participant was able to perform the activity at least 8 times. The single-leg balance was modified to a tandem stance if a participant was unable to perform a single leg stance. The participants were asked to complete two to three sets of 8-12 repetitions of each exercise, once daily. Participants performed the exercises twice/week in the lab under the supervision of a physiotherapist where they received support for exercise progression and problem solving any difficulties encountered with the exercises.

\section{Active phototherapy group}

The active phototherapy intervention group received phototherapy in addition to the education and exercise intervention twice weekly for 4 weeks. Participants were positioned in a comfortable and relaxed position on a therapy plinth. The invitalizer 3.0 workstation was used to apply High Intensity Physio Light $\left(\mathrm{HIPL}^{\mathrm{Tm}}\right)$ therapy (invitalize, Kitchener, Ontario, Canada). The phototherapy illuminated the affected knee for $20 \mathrm{~min}$. In the case of bilateral knee OA, the treatment was applied to both knees (20 min each). The knee was positioned approximately $2.54-7.62 \mathrm{~cm}$ from the phototherapy source with an intensity setting of $50 \mathrm{~mW} / \mathrm{cm}^{2}$. The invitalizer 3.0 workstation uses 5 panels of lights with a total emitter array area of $800 \mathrm{~cm}^{2}$. The 5 panels can be easily configured to allow the light source to be positioned consistently 2.54-7.62 $\mathrm{cm}$ from the skin over the anterior, medial, and lateral sides of the knee during application regardless of the size or shape of the participant's knee.

\section{Placebo group}

The control group received the same procedure as the active phototherapy intervention group (e.g., education and exercise), with the substitution of $20 \mathrm{~min}$ of placebo phototherapy (versus active phototherapy intervention) twice weekly for 4 weeks. The intensity of the HIPL ${ }^{\mathrm{rm}}$ therapy was set at $1 \mathrm{~mW} / \mathrm{cm}^{2}$ for the placebo group using the invitalizer 3.0 workstation (a dosage at which there was no therapeutic benefit expected, but the light was still be visible to the participant).

\section{Assessment and outcomes}

The feasibility outcomes described below were the primary outcomes of this pilot study. Secondary outcomes included the outcomes planned for the full trial. We determined the feasibility for collecting these outcomes and report them descriptively with no statistical comparisons made between groups.

\section{Feasibility outcomes}

Recruitment The feasibility of participant recruitment was determined by the overall recruitment rate. A full trial was considered feasible with our current recruitment methods if, during the pilot study, we were able to recruit 
three participants per week. This recruitment rate would allow us to exceed the estimated 168 participants needed for a fully powered trial in a 56-week recruitment period.

Retention Retention was assessed by attrition rate, with $<20 \%$ attrition at 3-month follow-up considered indicative of feasibility with our current protocol. We decided a priori that a full trial could be conducted with attrition rates of $<30 \%$, but with additional strategies to increase retention. This is based on evidence that $>20 \%$ attrition threatens trial validity [46].

Assessment procedures Feasibility of the assessment procedures were measured by completeness of data and duration of completing all outcome measures. As recommended for pilot studies, we set criteria a priori for acceptable completeness [47] and considered $>80 \%$ of all assessment items completed and a mean time for completion of $<60 \mathrm{~min}$ as acceptable.

Treatment fidelity Treatment fidelity was encouraged through training the physiotherapists delivering the program and by providing a treatment fidelity checklist to the research assistants/physiotherapists. Fidelity was measured through an audit of the fidelity checklist and reported as a proportion of intervention components (education, exercise, phototherapy) delivered in alignment with the protocol. An a-priori acceptable level of fidelity was considered $80 \%$ for each component of the intervention (education, exercise, and phototherapy) among participants that attend each visit.

\section{Participant characteristics}

The following measures were collected at baseline to describe the study population: age, sex, gender, duration of knee pain, current medications, comorbidities (using a standardized disease count to measure comorbidity [48, 49]), work status, annual household income, and number of other painful body sites.

\section{Primary outcome piloted for the full trial}

Pain during physical activity Pain during physical activity was measured using change in pain throughout a standardized physical activity, a measure called sensitivity to physical activity (SPA) in the literature [50-53]. SPA has been shown to explain unique variance in self-reported disability beyond that explained by static pain measure s[54], suggesting that there may be clinical value to measuring SPA as a distinct construct from pain at rest. In this study, SPA was measured using change in pain throughout a standardized 6-min walk test $(6 \mathrm{MWT})[50,53]$. Pain intensity was measured each minute throughout the 6MWT. The SPA index was calculated as the difference between the peak pain intensity during the 6MWT and baseline pain intensity at rest immediately prior to the test. SPA will be the primary outcome for the full trial.

\section{Secondary outcomes piloted for the full trial}

Pain severity Pain severity was measured using a $\mathrm{Nu}-$ meric Pain Rating Scale (NPRS) with anchors including no pain (0) to worst imaginable pain (10). Participants were asked to report their pain intensity at rest currently, on average over the last $24 \mathrm{~h}$, at its worst over the last $24 \mathrm{~h}$, and at its least over the last $24 \mathrm{~h}$. The pain severity was calculated as the mean of the four items. This combination of questions has been validated as a symptom severity index in the Brief Pain Inventory [55].

WOMAC physical function subscale Self-reported physical function was measured by the Western Ontario and McMaster Universities Osteoarthritis Index (WOMAC) $[34,56]$ physical function subscale (0 to 68) with higher scores indicating greater functional limitations. The WOMAC physical function subscale uses a series of questions about ability to perform functional tasks answered on a four-point Likert scale.

WOMAC pain subscale The WOMAC pain subscale $(0-20)$ was collected as a secondary pain intensity measure with higher scores indicating greater pain [56]. The WOMAC pain subscale asks participants to report their pain intensity during walking, stairs, in bed, sitting or lying, and standing on a four-point Likert scale. The WOMAC was chosen as the most appropriate physical function measure, but the WOMAC also includes pain and stiffness subscales. The pain subscale will be considered secondary to the pain severity index as a pain measure.

WOMAC stiffness subscale Stiffness was reported by participants on the WOMAC stiffness subscale (0-8) with higher scores indicating greater reports of stiffness [56]. The WOMAC stiffness subscale is a two-item scale asking participants to report their stiffness after waking up and later in the day on a four-item Likert Scale.

Adherence to prescribed exercise Adherence to prescribed exercise was measured using a self-reported daily $\log$ of all exercises prescribed throughout the 4-week treatment period. The log included whether each prescribed exercise was performed, the number of repetitions, and the number of sets of each exercise. The outcome is reported as proportion of prescribed exercises completed for each participant.

Global rating of change Global rating of change (GROC) was measured using an 11-point global rating of change 
scale (GROC) $(-5$ to +5$)$ as has been recommended in the literature for self-reported rating of change $[57,58]$.

Patient satisfaction Satisfaction was assessed using an 11-point scale with anchors of very dissatisfied $(-5)$ and very satisfied $(+5)$.

Adverse events Adverse events were recorded using an adverse events questionnaire that was reported consistently with reporting guidelines $[59,60]$ and asked (1) if the participant has experienced any adverse events as a result of any of the treatments received (yes/no); If any adverse events were reported, participants were asked the following: (2) what adverse events were experienced; (3) how long the event lasted (hours or days); and (4) how severe the adverse event was (0-10 scale).

Physical function performance A performance measure of physical function was the 6-min walk test (6MWT) [61, 62]. The 6MWT is a test of walking speed, aerobic capacity, and endurance. It involves walking continuously on a 30-m walkway for $6 \mathrm{~min}$. The score on the test was the distance walked. Distance walked was measured at the same time as the pain during physical activity measure.

Pressure pain threshold Pressure was applied using a digital handheld pressure algometer with a $1 \mathrm{~cm}^{2}$ rubber tip (Wagner Instruments, Texas). Pressure was applied to eight test sites in the peripatellar region, and one control site $5 \mathrm{~cm}$ distal to the tibial tuberosity, as described by Ardent-Neielson and colleagues [63]. Site 1: $2 \mathrm{~cm}$ distal to the inferior medial edge of patella; site 2: $2 \mathrm{~cm}$ distal to the inferior lateral edge of patella; site $3: 3 \mathrm{~cm}$ lateral to the mid point on the lateral edge of patella; site $4: 2 \mathrm{~cm}$ proximal to the superior lateral edge of patella; site $5: 2 \mathrm{~cm}$ proximal to the superior edge of patella; site $6: 2 \mathrm{~cm}$ proximal to the superior medial edge of patella; site $7: 3 \mathrm{~cm}$ medial to the mid point on the medial edge of patella; and site 8: at centre of patella. At each site, participants were asked to report the moment the pressure switched from comfortable pressure to slightly unpleasant pain [64]. The pressure pain threshold was recorded in Newtons $(\mathrm{N})$. The pressure pain threshold was calculated as the mean of measures across the 8 peripatellar sites and the pressure pain threshold at the standard tibialis anterior site was reported separately. Pressure pain threshold was piloted for the full trial in anticipation of including this as a process outcome to determine the relationships between pressure pain threshold (a measure of sensitivity to mechanical stimuli) and SPA.

\section{Timeline for assessment}

Participant characteristics were collected at baseline. The primary outcome (SPA) was measured before and after phototherapy at baseline, 2 weeks (mid-treatment period), and 4 weeks (end of treatment period). Self-reported secondary outcomes (pain severity and WOMAC [physical function, pain, and stiffness subscales]) were assessed at baseline, 2, 4, 8, 12, and 16 weeks (baseline, mid-treatment, end of treatment, 4-, 8-, and 12-week follow-ups). Adherence was measured daily throughout the 4-week treatment period through a patient log with data collected at each visit. Global rating of change and satisfaction were measured at all time points after the baseline assessment. $6 \mathrm{MWT}$ was measured before and after treatment at baseline, 2 weeks (mid-treatment), and 4 weeks (end of treatment). Pressure pain threshold was measured prior to phototherapy at baseline and 4 weeks (end of treatment).

Current pain intensity and adverse events were also measured immediately prior to phototherapy, immediately after phototherapy (before exercises), and immediately following completion of exercise to determine the immediate effects of the phototherapy and the combined phototherapy and exercise intervention.

\section{Data collection and management}

All baseline characteristics and outcomes were collected using Qualtrics online survey platform (Qualtrics, Provo, UT, USA, 2013). Assessments completed at baseline, 2 weeks, and 4 weeks were completed in-person with a blinded research assistant. Eight-, 12-, and 16-week assessments were completed through the online survey with individual links to the survey sent to participants and phone calls to remind participants to complete the surveys completed by the blinded research assistants. Multiple attempts were made to contact participants by phone or email for any participant who missed an assessment.

Analysis As recommended for pilot studies [47], feasibility outcomes are reported in relation to pre-specified feasibility criteria and outcomes piloted for the full trial are reported descriptively in aggregate and by group without statistical comparisons between groups. Means and standard deviations are reported for normal, continuous data. Medians and interquartile range are reported for non-normal data. Counts and percentages are reported for categorical data.

\section{Results}

Twenty adults with knee OA participated in this pilot study. The majority of participants were female (13/20) with a mean age of 63.95 (SD 9.27) years. Participant baseline characteristics are presented in Table 1.

\section{Feasibility outcomes}

\section{Recruitment rate}

Twenty participants were recruited over 3 weeks (6.7 participants/week) from two primary care sites. Recruitment was stopped when the targeted sample of 20 participants was reached. 
Table 1 Baseline characteristics of study participants

\begin{tabular}{|c|c|c|c|}
\hline Characteristics & Aggregated $(n=20)$ & Active phototherapy $(n=10)$ & Placebo control $(n=10)$ \\
\hline Age-years (mean, SD) & $63.95(9.27)$ & $60.60(10.35)$ & $67.30(7.01)$ \\
\hline \multicolumn{4}{|l|}{ Sex } \\
\hline Male $(n, \%)$ & $7(35)$ & $3(30)$ & $4(40)$ \\
\hline Female $(n, \%)$ & $13(65)$ & $7(70)$ & $6(60)$ \\
\hline \multicolumn{4}{|l|}{ Gender } \\
\hline $\operatorname{Man}(n, \%)$ & $7(35)$ & $3(30)$ & $4(40)$ \\
\hline Woman $(n, \%)$ & $13(65)$ & $7(70)$ & $6(60)$ \\
\hline \multicolumn{4}{|l|}{ Annual household income $(n, \%)$} \\
\hline$<\$ 20,000$ & $0(0)$ & $0(0)$ & $0(0)$ \\
\hline$\$ 20,000$ to $\$ 40,000$ & $7(35)$ & $4(40)$ & $3(30)$ \\
\hline$\$ 40,000$ to $\$ 60,000$ & $315)$ & $1(10)$ & $2(20)$ \\
\hline$\$ 60,000$ to $\$ 80,000$ & $2(10)$ & $1(10)$ & $1(10)$ \\
\hline$\$ 80,000$ to $\$ 100,000$ & $2(10)$ & $2(20)$ & $0(0)$ \\
\hline$>\$ 100,000$ & $6(30)$ & $2(20)$ & $440)$ \\
\hline \multicolumn{4}{|l|}{ Affected knee $(n, \%)$} \\
\hline Left & $16(80)$ & $8(80)$ & $8(80)$ \\
\hline Right & $4(20)$ & $2(20)$ & $2(20)$ \\
\hline Knee pain duration-years (mean, SD) & 7.38(9.25) & $7.15(10.21)$ & $7.60(8.74)$ \\
\hline Number of other body regions with pain (mean, SD) & $4.00(2.68)$ & $3.902 .64)$ & $4.10(2.85)$ \\
\hline \multicolumn{4}{|l|}{ NSAID usage in the past 3 months } \\
\hline Yes $(n, \%)$ & $7(44)$ & $3(42.90)$ & $4(44.44)$ \\
\hline No $(n, \%)$ & $9(56)$ & $4(57.10)$ & $5(55.56)$ \\
\hline \multicolumn{4}{|l|}{ Pain killer usage in the past three months } \\
\hline Yes $(n, \%)$ & $10(63)$ & $3(42.90)$ & $7(77.78)$ \\
\hline No $(n, \%)$ & $6(37)$ & $4(57.10)$ & $2(22.22)$ \\
\hline Number of comorbidities (median, IQR) & $0.80(1)$ & $0.70(1)$ & $0.90(1)$ \\
\hline \multicolumn{4}{|l|}{ Comorbidities $(n, \%)$} \\
\hline Heart attack & $1(5)$ & $0(0)$ & $1(10)$ \\
\hline Asthma & $315)$ & $0(0)$ & $3(30)$ \\
\hline Chronic obstructive pulmonary disease & $1(5)$ & $0(0)$ & $1(10)$ \\
\hline Diabetes & $735)$ & $3(30)$ & $4(40)$ \\
\hline Kidney problems secondary to diabetes & $1(5)$ & $1(10)$ & $0(0)$ \\
\hline Eye problems secondary to diabetes & $1(5)$ & $1(10)$ & $0(0)$ \\
\hline Poor kidney function & $1(5)$ & $1(10)$ & $0(0)$ \\
\hline Rheumatoid arthritis & $2(10)$ & $1(10)$ & $1(10)$ \\
\hline
\end{tabular}

$S D$ standard deviation, NSAID non-steroidal anti-inflammatory drug, IQR interquartile range

\section{Retention}

We achieved an attrition rate of $5 \%$ at the end of the study with $19 / 20$ participants completing the 12-week follow-up assessment at 16 weeks. The one person that did not complete the assessments at 16 weeks was from the active phototherapy group and was lost to follow-up due to severe illness. The CONSORT flow diagram extension for randomised pilot and feasibility trials is presented in Fig. 1 [60].

\section{Assessment procedures}

We achieved an $89 \%$ assessment completion rate across all time-points (89\% of all assessment items completed). Of the completed assessments, we had an over $99 \%$ item completion rate with very few missing items. The completion rate was $100 \%, 80 \%, 75 \%, 95 \%, 90 \%$, and $95 \%$ at the baseline, 2-, 4-, 8-, 12-, and 16-week assessments respectively. Importantly, the 2- and 4-week assessments relied on in-person appointments for completion of 


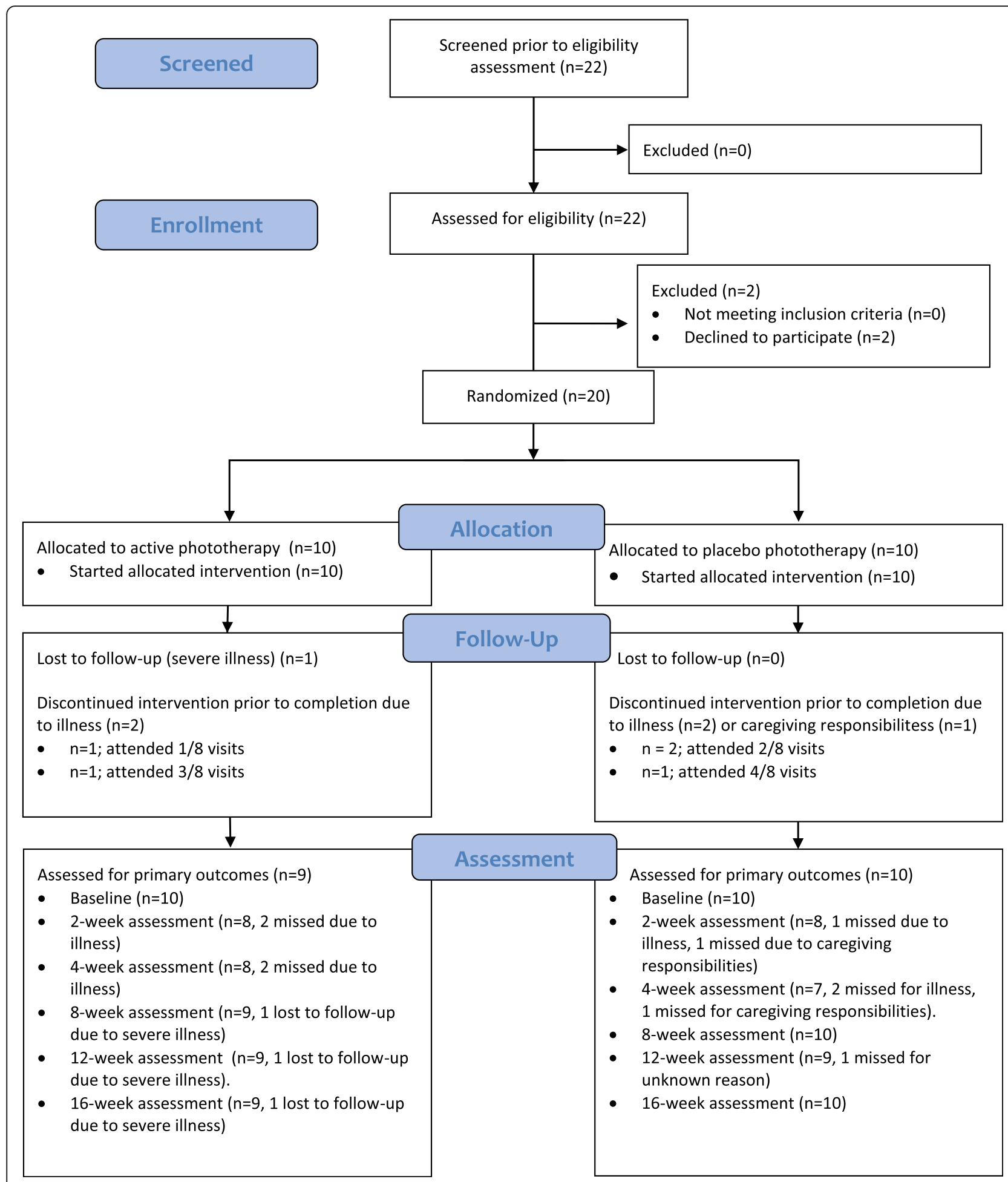

Fig. 1 CONSORT flow diagram extension to randomized pilot and feasibility studies

performance measures and psychophysical tests, whereas the 8-, 12-, and 16-week assessments were completed online or by phone. In addition to the participant who dropped out due to illness, the reasons reported for not completing the assessments were hospitalization $(n=1)$, caregiving responsibilities for a hospitalized spouse $(n=$ $1)$, mental health concerns $(n=1)$, and surgical complications $(n=1)$. These concerns were reported to interfere with in person assessments, but all of these four participants completed the final assessment through 
online data collection or by phone. Of the 19 completed follow-up assessments at 16 weeks, 18 completed them using the unique link provided by email and one requested to complete the assessment by phone.

The mean time to completion for outcome measures was $14.4 \mathrm{~min}$ (SD 5.7) at baseline, $5.9 \mathrm{~min}$ (SD 3.4 $\mathrm{min}$ ) at visit 4 assessment, and $5.7 \mathrm{~min}$ (SD 4.1) at visit 8 assessment. The mean time to completion at 8-week, 12week, and 16-week follow-up was difficult to calculate due to delays evident within the survey (i.e., people leaving the survey open or leaving and coming back). Removing any surveys that took over $30 \mathrm{~min}$ as outliers, the following mean times to completion were calculated: $10.7 \mathrm{~min}, 14.25 \mathrm{~min}$, and $21.2 \mathrm{~min}$ at $8-, 12-$, and $16-$ week follow-ups respectively.

\section{Treatment fidelity}

Fifteen of 20 people completed all eight in-person visits (8/10 from the active phototherapy group and 7/10 from the placebo group). One person completed 4 visits, one person completed 3 visits, two people completed 2 visits, and one person completed 1 visit. The audit of the fidelity checklist suggested that the active phototherapy intervention and placebo groups were delivered with $100 \%$ consistency with the planned protocol for all visits in which participants attended. Similarly, all participants received education and exercise consistent with the protocol in both groups.

\section{Outcomes piloted for a full trial}

We piloted the collection of primary and secondary outcomes for a full trial. Primary and secondary outcomes

Table 2 Aggregated outcome measure data (from active phototherapy intervention and placebo control groups) at all assessment time-points

\begin{tabular}{|c|c|c|c|c|c|c|c|c|c|c|c|c|c|c|c|c|c|c|}
\hline \multirow[t]{2}{*}{ Measures } & \multicolumn{3}{|c|}{ Baseline (week 0) } & \multicolumn{3}{|c|}{ Visit 4 (week2) } & \multicolumn{3}{|c|}{ Visit 8 (week 4) } & \multicolumn{3}{|c|}{$\begin{array}{l}\text { 4-week follow-up } \\
\text { (week 8) }\end{array}$} & \multicolumn{3}{|c|}{$\begin{array}{l}\text { 8-week follow-up } \\
\text { (week 12) }\end{array}$} & \multicolumn{3}{|c|}{$\begin{array}{l}\text { 12-week follow-up } \\
\text { (week 16) }\end{array}$} \\
\hline & $N$ & Mean & SD & $N$ & Mean & SD & $N$ & Mean & SD & $N$ & Mean & SD & $N$ & Mean & SD & $N$ & Mean & SD \\
\hline \multicolumn{19}{|c|}{ SPA (peak pain-baseline pain) } \\
\hline Pre & 20 & 2.17 & 1.18 & 16 & 0.81 & 0.75 & 15 & 0.60 & 1.24 & - & - & - & - & - & - & - & - & - \\
\hline Post & 20 & 1.33 & 1.15 & 16 & 0.56 & 0.81 & 15 & 0.53 & 1.13 & - & - & - & - & - & - & - & - & - \\
\hline \multicolumn{19}{|l|}{ Pain severity index } \\
\hline Worst & 20 & 4.75 & 2.15 & 16 & 3.37 & 2.27 & 15 & 3.00 & 1.81 & 19 & 3.11 & 2.73 & 18 & 3.17 & 2.77 & 19 & 3.11 & 2.75 \\
\hline At least & 20 & 1.70 & 1.63 & 16 & 0.69 & 1.07 & 15 & 0.20 & 0.41 & 19 & 1.37 & 2.45 & 18 & 1.39 & 2.42 & 19 & 1.16 & 2.36 \\
\hline Average & 20 & 3.55 & 1.50 & 16 & 1.81 & 1.47 & 15 & 1.20 & 1.01 & 19 & 2.42 & 2.36 & 18 & 2.17 & 2.36 & 19 & 2.00 & 2.33 \\
\hline Right now & 20 & 2.95 & 2.01 & 16 & 1.81 & 1.94 & 15 & 0.93 & 1.62 & 19 & 1.63 & 2.81 & 18 & 1.83 & 2.87 & 19 & 1.79 & 3.01 \\
\hline Index & 20 & 3.24 & 1.61 & 16 & 1.92 & 1.45 & 15 & 1.33 & 0.90 & 19 & 2.13 & 2.53 & 18 & 2.14 & 2.52 & 19 & 2.01 & 2.48 \\
\hline \multicolumn{19}{|l|}{ WOMAC } \\
\hline Pain & 20 & 6.30 & 2.34 & 16 & 4.31 & 2.44 & 15 & 3.60 & 2.35 & 19 & 4.58 & 3.77 & 18 & 5.00 & 4.11 & 19 & 4.53 & 4.03 \\
\hline Stiffness & 20 & 3.10 & 1.70 & 16 & 2.25 & 1.53 & 15 & 1.67 & 1.11 & 19 & 2.89 & 1.62 & 18 & 2.67 & 1.97 & 19 & 1.79 & 1.75 \\
\hline Physical Function & 20 & 22.95 & 9.72 & 16 & 15.81 & 10.52 & 15 & 9.913 & 6.81 & 19 & 17.32 & 16.66 & 18 & 17.83 & 16.99 & 19 & 13.63 & 14.79 \\
\hline Total & 20 & 32.35 & 12.00 & 16 & 22.38 & 13.63 & 15 & 14.40 & 9.32 & 19 & 24.79 & 21.11 & 18 & 25.50 & 22.49 & 19 & 19.95 & 20.14 \\
\hline GROC Function & - & - & - & 16 & 7.19 & 1.05 & 15 & 8.27 & 1.44 & 19 & 7.42 & 2.01 & 18 & 7.78 & 2.16 & 19 & 8.32 & 1.67 \\
\hline GROC Pain & - & - & - & 16 & 6.87 & 1.26 & 15 & 8.00 & 1.65 & 19 & 7.21 & 2.07 & 18 & 7.61 & 2.09 & 19 & 7.89 & 1.91 \\
\hline Satisfaction & - & - & - & 16 & 10.25 & 1.29 & 15 & 10.33 & 0.76 & 19 & 9.84 & 1.97 & 18 & 9.56 & 2.50 & 19 & 9.84 & 1.74 \\
\hline \multicolumn{19}{|l|}{ 6MWT (m) } \\
\hline Pre & 20 & 356.88 & 100.58 & 16 & 421.61 & 82.18 & 15 & 450.61 & 72.17 & - & - & - & - & - & - & - & - & - \\
\hline Post & 20 & 360.20 & 97.29 & 16 & 417.77 & 80.83 & 15 & 465.18 & 82.50 & - & - & - & - & - & - & - & - & - \\
\hline \multicolumn{19}{|c|}{ Pressure pain threshold-mean of eight peripatellar sites $(N)$} \\
\hline Most painful knee & 20 & 49.18 & 34.00 & - & - & - & 15 & 59.03 & 27.19 & - & - & - & - & - & - & - & - & - \\
\hline Least painful knee & 20 & 52.40 & 34.94 & - & - & - & 15 & 64.76 & 47.72 & - & - & - & - & - & - & - & - & - \\
\hline \multicolumn{19}{|c|}{ Pressure pain threshold - standard site over tibialis anterior $(N)$} \\
\hline Most painful knee & 20 & 42.68 & 28.68 & - & - & - & 15 & 47.37 & 25.02 & - & - & - & - & - & - & - & - & - \\
\hline Least painful knee & 20 & 43.73 & 27.08 & - & - & - & 15 & 56.27 & 23.44 & - & - & - & - & - & - & - & - & - \\
\hline
\end{tabular}


are reported in aggregate form in Table 2. The standard deviations from these results will help to inform sample size calculations for a full trial.

The results for the piloted primary outcomes for the active phototherapy intervention group are presented for the active phototherapy group in Table 3 . The results for the piloted primary outcomes for the placebo control group are presented for the placebo control group in Table 4.

The results for adherence to prescribed exercise are presented in Table 5. The mean overall adherence to prescribed exercise over the 4-week treatment period was $93.60 \%$ (SD 9.36) across all visits among both treatment groups.

The results for current pain intensity and adverse events immediately before and after treatment are presented in Table 6. Pre-post differences in pain intensity from before to after the phototherapy for aggregated, active phototherapy, and placebo control groups are presented in Table 7 . There were no adverse events reported in either group.

\section{Discussion}

Our results suggest that a future randomized controlled trial evaluating the effectiveness of active phototherapy intervention versus placebo control in a population with knee OA is feasible. We achieved a recruitment rate of 6.7 participants/week over 3 weeks from two primary care sites. At this rate, a fully powered randomized controlled trial with 168 participants could be recruited in approximately 25 weeks, which would provide $90 \%$ power to detect a moderate effect size difference between groups with an alpha of 0.05 . The retention rate of $95 \%$ at the end of study and overall assessment completion rate of $89 \%$ also exceeded our a priori target for

Table 3 Outcome measure data from active phototherapy intervention group at all assessment time-points

\begin{tabular}{|c|c|c|c|c|c|c|c|c|c|c|c|c|c|c|c|c|c|c|}
\hline \multirow[t]{2}{*}{ Measures } & \multicolumn{3}{|c|}{ Baseline (week 0) } & \multicolumn{3}{|c|}{ Visit 4 (week2) } & \multicolumn{3}{|c|}{ Visit 8 (week 4) } & \multicolumn{3}{|c|}{$\begin{array}{l}\text { 4-week follow-up } \\
\text { (week 8) }\end{array}$} & \multicolumn{3}{|c|}{$\begin{array}{l}\text { 8-week follow-up } \\
\text { (week 12) }\end{array}$} & \multicolumn{3}{|c|}{$\begin{array}{l}\text { 12-week follow-up } \\
\text { (week 16) }\end{array}$} \\
\hline & $N$ & Mean & SD & $N$ & Mean & SD & $N$ & Mean & SD & $N$ & Mean & SD & $N$ & Mean & SD & $N$ & Mean & SD \\
\hline \multicolumn{19}{|c|}{ SPA (peak pain-baseline pain) } \\
\hline Pre & 10 & 2.00 & 1.41 & 8 & 0.75 & 0.89 & 8 & 0.50 & 1.07 & - & - & - & - & - & - & - & - & - \\
\hline Post & 10 & 1.30 & 1.34 & 8 & 0.50 & 0.93 & 8 & 0.38 & 1.06 & - & - & - & - & - & - & - & - & - \\
\hline \multicolumn{19}{|l|}{ Pain severity } \\
\hline Worst & 10 & 4.20 & 2.04 & 8 & 2.88 & 2.42 & 8 & 3.00 & 1.77 & 9 & 1.67 & 1.50 & 8 & 1.75 & 2.32 & 9 & 2.44 & 3.09 \\
\hline At least & 10 & 1.70 & 1.49 & 8 & 0.25 & 0.46 & 8 & 0.25 & 0.46 & 9 & 0.22 & 0.44 & 8 & 0.25 & 0.46 & 9 & 0.44 & 1.01 \\
\hline Average & 10 & 3.30 & 1.42 & 8 & 1.38 & 1.06 & 8 & 1.13 & 0.84 & 9 & 1.33 & 1.12 & 8 & 0.88 & 0.99 & 9 & 1.22 & 1.72 \\
\hline Right now & 10 & 3.10 & 2.13 & 8 & 2.13 & 2.59 & 8 & 0.38 & 0.52 & 9 & 0.11 & 0.33 & 8 & 0.75 & 1.75 & 9 & 0.44 & 3.28 \\
\hline Index & 10 & 3.08 & 1.95 & 8 & 1.66 & 2.03 & 8 & 1.88 & 1.49 & 9 & 0.83 & 1.16 & 8 & 0.91 & 1.57 & 9 & 1.39 & 2.46 \\
\hline \multicolumn{19}{|l|}{ WOMAC } \\
\hline Pain & 10 & 5.50 & 2.12 & 8 & 3.25 & 2.25 & 8 & 3.13 & 2.10 & 9 & 2.44 & 1.42 & 8 & 2.38 & 1.69 & 9 & 2.78 & 2.73 \\
\hline Stiffness & 10 & 3.20 & 1.75 & 8 & 1.50 & 1.20 & 8 & 1.38 & 1.06 & 9 & 2.11 & 1.27 & 8 & 1.62 & 1.51 & 9 & 0.78 & 0.83 \\
\hline Physical Function & 10 & 20.70 & 11.82 & 8 & 10.25 & 7.36 & 8 & 7.75 & 5.95 & 9 & 11.00 & 9.15 & 8 & 9.63 & 9.61 & 9 & 7.56 & 7.47 \\
\hline Total & 10 & 29.40 & 14.29 & 8 & 15.00 & 10.23 & 8 & 12.25 & 8.01 & 9 & 15.56 & 10.85 & 8 & 13.63 & 11.43 & 9 & 11.11 & 10.42 \\
\hline GROC Function & - & - & - & 8 & 7.38 & 1.30 & 8 & 8.63 & 1.85 & 9 & 8.33 & 1.32 & 8 & 8.87 & 1.25 & 9 & 9.11 & 1.45 \\
\hline GROC Pain & - & - & - & 8 & 7.25 & 1.39 & 8 & 8.25 & 1.83 & 9 & 8.22 & 1.39 & 8 & 8.63 & 1.30 & 9 & 8.67 & 1.66 \\
\hline Satisfaction & - & - & - & 8 & 10.88 & 0.35 & 8 & 11.00 & 0.00 & 9 & 10.22 & 1.99 & 8 & 10.38 & 1.41 & 9 & 10.44 & 1.33 \\
\hline \multicolumn{19}{|l|}{ 6MWT (m) } \\
\hline Pre & 10 & 391.78 & 103.98 & 8 & 449.85 & 63.53 & 8 & 469.57 & 50.71 & - & - & - & - & - & - & - & - & - \\
\hline Post & 10 & 402.62 & 88.99 & 8 & 456.09 & 64.78 & 8 & 489.27 & 62.21 & - & - & - & - & - & - & - & - & - \\
\hline \multicolumn{19}{|c|}{ Pressure pain threshold-mean of periopaterllar sites $(N)$} \\
\hline Most painful knee & 10 & 46.49 & 31.46 & - & - & - & 8 & 57.68 & 29.82 & - & - & - & - & - & - & - & - & - \\
\hline Least painful knee & 10 & 52.09 & 40.40 & - & - & - & 8 & 56.70 & 27.91 & - & - & - & - & - & - & - & - & - \\
\hline \multicolumn{19}{|c|}{ Pressure pain threshold-standard site over tibialis anterior $(N)$} \\
\hline Most painful knee & 10 & 47.7 & 35.83 & - & - & - & 8 & 53.38 & 25.52 & - & - & - & - & - & - & - & - & - \\
\hline Least painful knnee & 10 & 47.25 & 34.92 & - & - & - & 8 & 49.56 & 26.77 & - & - & - & - & - & - & - & - & - \\
\hline
\end{tabular}


Table 4 Outcome measure data from placebo control group at all assessment time-points

\begin{tabular}{|c|c|c|c|c|c|c|c|c|c|c|c|c|c|c|c|c|c|c|}
\hline \multirow[t]{2}{*}{ Measures } & \multicolumn{3}{|c|}{$\begin{array}{l}\text { Baseline (week } \\
\text { 0) }\end{array}$} & \multicolumn{3}{|c|}{ Visit 4 (week2) } & \multicolumn{3}{|c|}{ Visit 8 (week 4) } & \multicolumn{3}{|c|}{$\begin{array}{l}\text { 4-week follow-up } \\
\text { (week 8) }\end{array}$} & \multicolumn{3}{|c|}{$\begin{array}{l}\text { 8-week follow-up } \\
\text { (week 12) }\end{array}$} & \multicolumn{3}{|c|}{$\begin{array}{l}\text { 12-week follow-up } \\
\text { (week 16) }\end{array}$} \\
\hline & $N$ & Mean & SD & $N$ & Mean & SD & $N$ & Mean & SD & $N$ & Mean & SD & $N$ & Mean & SD & $N$ & Mean & SD \\
\hline \multicolumn{19}{|c|}{ SPA (peak pain-baseline pain) } \\
\hline Pre & 10 & 2.35 & 0.94 & 8 & 0.50 & 0.76 & 7 & 0.00 & 0.16 & - & - & - & - & - & - & - & - & - \\
\hline Post & 10 & 1.35 & 1.00 & 8 & 0.63 & 0.74 & 7 & 0.57 & 1.27 & - & - & - & - & - & - & - & - & - \\
\hline \multicolumn{19}{|l|}{ Pain severity } \\
\hline Worst & 10 & 5.30 & 2.21 & 8 & 3.87 & 2.17 & 7 & 3.00 & 2.00 & 10 & 4.40 & 2.99 & 10 & 4.30 & 2.67 & 10 & 3.70 & 2.41 \\
\hline At least & 10 & 1.70 & 1.83 & 8 & 1.13 & 1.36 & 7 & 0.14 & 0.38 & 10 & 2.40 & 3.06 & 10 & 2.30 & 2.98 & 10 & 1.80 & 3.05 \\
\hline Average & 10 & 3.80 & 1.62 & 8 & 2.25 & 1.75 & 7 & 1.29 & 1.25 & 10 & 3.40 & 2.80 & 10 & 3.20 & 2.66 & 10 & 2.70 & 2.67 \\
\hline Right now & 10 & 2.80 & 1.99 & 8 & 1.50 & 1.07 & 7 & 1.57 & 2.23 & 10 & 3.00 & 3.37 & 10 & 2.70 & 3.37 & 10 & 2.10 & 2.89 \\
\hline Index & 10 & 3.4 & 2.28 & 8 & 2.19 & 1.89 & 7 & 1.50 & 1.86 & 10 & 3.30 & 3.03 & 10 & 3.1 & 2.92 & 10 & 2.6 & 2.75 \\
\hline \multicolumn{19}{|l|}{ WOMAC } \\
\hline Pain & 10 & 7.10 & 2.38 & 8 & 5.38 & 2.26 & 7 & 4.14 & 2.67 & 10 & 6.50 & 4.25 & 10 & 7.10 & 4.33 & 10 & 6.10 & 4.48 \\
\hline Stiffness & 10 & 3.00 & 0.94 & 8 & 3.00 & 1.51 & 7 & 2.00 & 1.16 & 10 & 3.60 & 1.65 & 10 & 3.50 & 1.96 & 10 & 2.70 & 1.89 \\
\hline Physical Function & 10 & 25.20 & 6.96 & 8 & 21.38 & 10.03 & 7 & 10.71 & 7.85 & 10 & 23.00 & 20.13 & 10 & 24.40 & 19.12 & 10 & 19.10 & 17.84 \\
\hline Total & 10 & 35.30 & 8.98 & 8 & 29.75 & 13.01 & 7 & 16.86 & 10.72 & 10 & 33.10 & 25.00 & 10 & 35.00 & 25.07 & 10 & 27.90 & 23.81 \\
\hline GROC Function & - & - & - & 8 & 7.00 & 0.76 & 7 & 7.86 & 0.69 & 10 & 6.60 & 2.22 & 10 & 6.90 & 2.38 & 10 & 7.60 & 1.58 \\
\hline GROC Pain & - & - & - & 8 & 6.50 & 1.07 & 7 & 7.71 & 1.50 & 10 & 6.30 & 2.14 & 10 & 6.80 & 2.30 & 10 & 7.20 & 1.93 \\
\hline Satisfaction & - & - & - & 8 & 9.62 & 1.60 & 7 & 9.57 & 0.98 & 10 & 9.50 & 2.01 & 10 & 8.90 & 3.04 & 10 & 9.30 & 1.95 \\
\hline \multicolumn{19}{|l|}{$6 \mathrm{MWT}(\mathrm{m})$} \\
\hline Pre & 10 & 321.97 & 88.53 & 8 & 393.37 & 92.82 & 7 & 428.95 & 90.15 & - & - & - & - & - & - & - & - & - \\
\hline Post & 10 & 317.77 & 89.80 & 8 & 379.44 & 80.30 & 6 & 433.05 & 100.56 & - & - & - & - & - & - & - & - & - \\
\hline \multicolumn{19}{|c|}{ Pressure pain threshold-mean of peripatellar sites $(N)$} \\
\hline Most tender knee & 10 & 51.89 & 36.27 & - & - & - & 7 & 60.29 & 25.01 & - & - & - & - & - & - & - & - & - \\
\hline Least tender knee & 10 & 52.70 & 28.57 & - & - & - & 7 & 73.98 & 62.05 & - & - & - & - & - & - & - & - & - \\
\hline \multicolumn{19}{|c|}{ Pressure pain threshold - standard site over tibialis anterior $(N)$} \\
\hline Most tender knee & 10 & 38.10 & 18.87 & - & - & - & 7 & 42.64 & 12.52 & - & - & - & - & - & - & - & - & - \\
\hline Least tender knee & 10 & 40.20 & 16.10 & - & - & - & 7 & 48.50 & 18.93 & - & - & - & - & - & - & - & - & - \\
\hline
\end{tabular}

SPA sensitivity to physical activity, WOMAC Western Ontario and McMaster Universities Osteoarthritis Index, 6MWT 6-minute walk test, GROC global rating of change, $N$ Newtons

Table 5 Self-reported adherence to prescribed exercise program

\begin{tabular}{|c|c|c|c|c|c|c|}
\hline \multirow[t]{2}{*}{$\begin{array}{l}\text { Component of exercise } \\
\text { intervention }\end{array}$} & \multicolumn{2}{|c|}{$\begin{array}{l}\text { Aggregated, } n=15 \text { (\% of } \\
\text { prescribed exercises completed } \\
\text { over 4-week treatment period) }\end{array}$} & \multicolumn{2}{|c|}{$\begin{array}{l}\text { Active phototherapy, } n=8 \text { (\% of } \\
\text { prescribed exercises completed } \\
\text { over 4-week treatment period) }\end{array}$} & \multicolumn{2}{|c|}{$\begin{array}{l}\text { Placebo, } n=7 \text { (\% of prescribed } \\
\text { exercises completed over 4-week } \\
\text { treatment period) }\end{array}$} \\
\hline & Mean & SD & Mean & SD & Mean & SD \\
\hline Chair squats & 95.44 & 6.85 & 94.34 & 5.07 & 96.70 & 8.72 \\
\hline Step-ups & 94.91 & 5.95 & 94.34 & 5.07 & 95.56 & 7.20 \\
\hline Lunge & 94.38 & 6.85 & 93.82 & 5.57 & 95.01 & 8.51 \\
\hline Resisted knee flexion & 94.91 & 6.61 & 94.34 & 5.07 & 95.56 & 8.43 \\
\hline Single leg stance & 95.68 & 5.02 & 94.34 & 5.07 & 97.21 & 4.88 \\
\hline $\begin{array}{l}\text { Knee flexion/extension } \\
\text { range of motion }\end{array}$ & 95.94 & 4.71 & 94.34 & 5.07 & 97.76 & 3.83 \\
\hline Walking program & 83.97 & 17.67 & 91.36 & 6.59 & 75.53 & 22.84 \\
\hline Overall & 93.60 & 9.36 & 93.84 & 5.19 & 93.33 & 12.60 \\
\hline
\end{tabular}


Table 6 Immediate effects on pain intensity and adverse effects for aggregated, active phototherapy, and placebo control groups

\begin{tabular}{|c|c|c|c|c|c|c|c|c|c|c|c|c|}
\hline \multicolumn{2}{|c|}{ Visit number ${ }^{a}$} & \multicolumn{9}{|c|}{ Currnet pain intensity ( 0 to 10 ) } & \multicolumn{2}{|c|}{$\begin{array}{l}\text { Adverse effects-number of participants } \\
\text { reporting "yes" to the question "did you } \\
\text { experience any adverse effects or side effects } \\
\text { during the treatment today?" }\end{array}$} \\
\hline & & \multicolumn{3}{|c|}{ Aggregated } & \multicolumn{3}{|c|}{$\begin{array}{l}\text { Active } \\
\text { phototherapy }\end{array}$} & \multicolumn{3}{|c|}{$\begin{array}{l}\text { Placebo } \\
\text { control }\end{array}$} & \multirow[t]{2}{*}{ Active Phototherapy } & \multirow[t]{2}{*}{ Placebo control } \\
\hline & & $N$ & Mean & SD & $N$ & Mean & SD & $N$ & Mean & SD & & \\
\hline \multirow[t]{3}{*}{ Visit 2} & Pre-phototherapy & 19 & 1.68 & 2.11 & 9 & 1.33 & 1.23 & 10 & 2.00 & 2.71 & 0 & 0 \\
\hline & Post-phototherapy & 19 & 0.84 & 1.30 & 9 & 0.78 & 1.09 & 10 & 0.90 & 1.52 & 0 & 0 \\
\hline & Post-phototherapy and exercise & 19 & 1.68 & 1.53 & 9 & 1.56 & 1.33 & 10 & 1.80 & 1.75 & 0 & 0 \\
\hline \multirow[t]{3}{*}{ Visit 3} & Pre-phototherapy & 18 & 2.06 & 2.53 & 9 & 1.78 & 2.54 & 9 & 2.33 & 2.65 & 0 & 0 \\
\hline & Post-phototherapy & 18 & 1.22 & 2.05 & 9 & 1.11 & 2.26 & 9 & 1.33 & 1.94 & 0 & 0 \\
\hline & Post-phototherapy and exercise & 18 & 1.50 & 1.58 & 9 & 1.22 & 1.79 & 9 & 1.78 & 1.39 & 0 & 0 \\
\hline \multirow[t]{3}{*}{ Visit 5} & Pre-phototherapy & 15 & 1.20 & 1.37 & 8 & 1.25 & 1.28 & 7 & 0.43 & 0.79 & 0 & 0 \\
\hline & Post-phototherapy & 15 & 0.40 & 0.74 & 8 & 0.38 & 0.52 & 7 & 0.00 & 0.00 & 0 & 0 \\
\hline & Post-phototherapy and exercise & 15 & 1.07 & 1.22 & 8 & 0.50 & 1.07 & 7 & 0.86 & 1.22 & 0 & 0 \\
\hline \multirow[t]{3}{*}{ Visit 6} & Pre-phototherapy & 15 & 0.53 & 0.64 & 8 & 0.38 & 0.52 & 7 & 0.71 & 0.76 & 0 & 0 \\
\hline & Post-phototherapy & 15 & 0.33 & 0.82 & 8 & 0.50 & 1.07 & 7 & 0.14 & 0.38 & 0 & 0 \\
\hline & Post-phototherapy and exercise & 15 & 0.53 & 0.92 & 8 & 0.50 & 0.76 & 7 & 0.57 & 1.13 & 0 & 0 \\
\hline \multirow[t]{3}{*}{ Visit 7} & Pre-phototherapy & 15 & 0.47 & 0.64 & 8 & 0.25 & 0.46 & 7 & 0.71 & 0.76 & 0 & 0 \\
\hline & Post-phototherapy & 15 & 0.13 & 0.35 & 8 & 0.13 & 0.35 & 7 & 0.14 & 0.38 & 0 & 0 \\
\hline & Post-phototherapy and exercise & 15 & 0.87 & 1.25 & 8 & 0.38 & 0.52 & 7 & 1.43 & 1.62 & 0 & 0 \\
\hline
\end{tabular}

Visits 1, 4, and 8 did not include pre-post measures for pain intensity. On visits 1, 4, and 8, pain intensity was measused prior to the sensitivity to physical activity (SPA) test which resulted in an increase in pain intensity immediately prior to phototherapy for most participants. Thus, the results of visits 1 , 4 , and 8 could not be pooled with the results from the other visits

proceeding with the full trial. Finally, the pilot study demonstrated strong treatment fidelity with $100 \%$ of treatment components delivered in alignment with the study protocol across all completed visits.

One challenge experienced in this trial was lower assessment completion rates for in-person assessments at 2 and 4 weeks ( $80 \%$ and $75 \%$ completion respectively) in comparison to online follow-up assessments in which $90 \%$ or greater completion rates were maintained. This is important, especially given that the primary outcome of the future RCT is a measure of pain during a standardized
6MWT, which requires in person testing. The challenges in attending in person assessments was largely due to the medical complexity of the sample participating in the study. One person dropped out of the study prior to the 2week assessment, reporting a severe illness that was not directly related to their OA. The other four participants who did not complete the 4-week assessment all missed their appointments due to comorbid health conditions affecting them or their partner. The high completion rate of online assessments despite these ongoing challenges for participants was an indicator of commitment of

Table 7 Change in pain intensity pre-post phototherapy for aggregated, active phototherapy, and placebo control groups

\begin{tabular}{|c|c|c|c|c|c|c|c|c|c|}
\hline \multirow[t]{3}{*}{ Visit number $^{a}$} & \multicolumn{9}{|c|}{ Change in pain intensity pre-post phototherapy (0 to 10$)$} \\
\hline & \multicolumn{3}{|c|}{ Aggregated } & \multicolumn{3}{|c|}{ Active phototherapy } & \multicolumn{3}{|c|}{ Placebo control } \\
\hline & $N$ & Mean & SD & $N$ & Mean & SD & $N$ & Mean & SD \\
\hline Visit 2 & 19 & -0.84 & 1.17 & 9 & -0.56 & 0.88 & 10 & -1.10 & 1.37 \\
\hline Visit 3 & 18 & -0.83 & 1.34 & 9 & -0.67 & 1.32 & 9 & -1.00 & 1.41 \\
\hline Visit 5 & 15 & -0.80 & 1.37 & 8 & -1.13 & 1.73 & 7 & -0.43 & 0.79 \\
\hline Visit 6 & 15 & -0.20 & 1.08 & 8 & 0.13 & 1.25 & 7 & -0.57 & 0.79 \\
\hline Visit 7 & 15 & -0.33 & 0.49 & 8 & -0.13 & 0.35 & 7 & -0.57 & 0.53 \\
\hline Mean of means for Visits $2,3,5,6$, and 7 & - & -0.60 & 0.31 & - & -0.47 & 0.49 & - & -0.73 & 0.30 \\
\hline
\end{tabular}

aisits 1, 4, and 8 did not include pre-post measures for pain intensity. On visits 1, 4, and 8, pain intensity was measused prior to the sensitivity to physical activity (SPA) test which resulted in an increase in pain intensity immediately prior to phototherapy for most participants. Thus, the results of visits 1 , 4 , and 8 could not be pooled with the results from the other visits 
participants to participating in the study. Therefore, we feel confident that the information and consent process effectively informed participants and that changing the information and consent process would not improve the in-person completion rate. We also discussed how changes to the inclusion or exclusion criteria may have helped to identify participants with multiple comorbidities more likely to experience health complications that could interfere with participation. However, all investigators agreed that the strength of this study was the recruitment of participants through primary care settings to best represent the population of people with OA seeking care, who often experience multiple comorbidities [65-70].

The finding of lower rates of assessment completion at in-person visits will inform the fully powered trial in three ways. (1) We will aim to recruit from primary care sites where they have the physical space available to complete the 6MWT onsite. By offering participants with barriers to attending in person at the university lab the opportunity to complete the assessment in conjunction with another healthcare appointment at their primary care site, we may be able to help participants overcome geographic barriers to participation which may increase our rate of competition of the primary outcome. This is a strategy the research team has implemented successfully in other trials. (2) We will follow-up multiple times via email and telephone with participants who are unable to attend inperson assessments to ensure that we still achieve high rates of completion of self-report measures at those timepoints, even if we are not able to capture the performance measures. 3) We will update our sample size calculation in order to allow for up to $25 \%$ dropout. These strategies will be implemented for a fully powered trial to address the challenges of achieving high assessment completion rate for the in-person assessments, while maintaining the strength of the study in representing the population of people with OA who are seeking care to facilitate generalizability of the results.

This study has important limitations that must be acknowledged. First, although our primary outcome piloted for a future trial (SPA) has demonstrated to be crosssectionally related to disability in adults with knee OA [50], the SPA measure we used measures change in pain during a walking task only. Change in pain during walking may not fully capture the complexity of pain during physical activity reported by people living with knee OA. Second, we did not control for co-intervention (e.g., intra-articular joint injections) among participants in this research. As such, it is possible that co-interventions may have been a confounding factor which impacted the results. We plan to use a pragmatic design, but will plan a sensitivity analysis for the full trial to assess whether the effect of the intervention is influenced by cointerventions. Third, our inclusion criteria indicated that participants had to receive a diagnosis of knee OA from a physician. We did not specify that participants needed to be radiographically diagnosed in order to meet inclusion criteria. As such, it is possible that some participants in this research could have had knee pain diagnosed as knee OA, but without radiographic evidence of knee OA. Our fully powered trial will include radiographic evidence within the inclusion criteria.

\section{Conclusion}

The results of this pilot study suggest that it is feasible to implement a fully powered randomized controlled trial to evaluate the effectiveness of an active phototherapy intervention versus placebo at reducing pain during movement in a population with knee OA.

\section{Abbreviations}

RCT: Randomized controlled trial; OA: Osteoarthritis; TENS: Transcutaneous electrical nerve stimulation; $\mathrm{mW} / \mathrm{cm}^{2}$ : Milliwatt per square centimeter; SPA: Sensitivity to physical activity; 6MWT: Six-minute walk test; NPRS: Numeric pain rating scale; BPI: Brief Pain Inventory; WOMAC: Western Ontario and McMaster Universities Ostoearthritis Index; GROC: Global rating of change; N: Newtons; SD: Standard deviation; NSAID: Non-steroidal antiinflammatory drug; IQR: Interquartile range

\section{Acknowledgements}

The authors wish to thank all participants who volunteered to participate in this research. The authors also thank the physicians, staff, and administrators at Oak Tree Family Health Center and Willow Family Health from Maple

Family Health Team who supported this study with participant recruitment.

\section{Authors' contributions}

$K V, A A B, M B C$, and $K V$ were involved with data collection and analysis; contributed to writing the manuscript; and approved the final submitted manuscript. JM conceptualized the study objectives, led the team in protocol development and securing funding; provided oversight for data collection, management and analysis; contributed to writing the manuscript; and approved the final submitted manuscript.

\section{Funding}

This research was supported by an Ontario Centers of Excellence (OCE)Voucher for Innovation and Productivity (VIP, \#28464), supporting partnerships between Ontario Corporation and publicly funded postsecondary institutions. The funding for the VIP included matched funds from OCE $(\$ 20,000$ grant) and invitalize ( $\$ 5,000$ cash $+\$ 15,000$ in-kind support). The in-kind contribution of invitalize included the provision the phototherapy equipment, expert consulting services in phototherapy, and programming support for implementation of the randomized controlled trial protocol. The invitalize corporation did not otherwise have any role in study design or in collection, analysis and interpretation of the data, or in writing up of this paper. Kyle Vader was supported by a Frederick Banting and Charles Best Canada Graduate Scholarship (CGS-D) awarded by the Canadian Institutes of Health Research (CIHR) (Funding Reference Number: GSD-157891). Abey Bekele Abebe and Mulugeta Bayisa Chala were supported by the Mastercard Foundations Scholars Program. Outside of the programming of the phototherapy device, the funders did not have a role in the study design or in the collection, analysis, interpretation, or writing up of the data.

\section{Availability of data and materials}

The datasets used and/or analyzed in this research are available from the corresponding author upon request.

\section{Ethics approval and consent to participate}

Ethical approval was obtained from the Queen's University Health Sciences and Affiliated Teaching Hospitals Research Ethics Board in Kingston, Ontario, Canada (Reference Number: 6022509). All participants provided written informed consent prior to participating in this research. 


\section{Consent for publication}

Not applicable.

\section{Competing interests}

The authors declare they have no competing interests. The funding for this research came in part from invitalize, who would benefit from positive results in this study. However, invitalize remained at arms length from the investigators throughout data collection, analysis, writing, and publication.

\section{Author details}

'School of Rehabilitation Therapy, Queen's University, Kingston, ON, Canada ${ }^{2}$ Chronic Pain Clinic, Kingston Health Sciences Centre, Kingston, ON, Canada.

\section{Received: 11 March 2020 Accepted: 16 November 2020} Published online: 26 November 2020

\section{References}

1. Vos T, Flaxman AD, Naghavi M, Lozano R, Michaud C, Ezzati M, Shibuya K, Salomon JA, Abdalla S, Aboyans V, Abraham J. Years lived with disability (YLDs) for 1160 sequelae of 289 diseases and injuries 1990-2010: a systematic analysis for the Global Burden of Disease Study 2010. Lancet. 2012;380(9859):2163-96

2. Woolf AD, Pfleger B. Burden of major musculoskeletal conditions. Bull World Health Organ. 2003:81:646-56.

3. Palazzo C, Ravaud JF, Papelard A, Ravaud P, Poiraudeau S. The burden of musculoskeletal conditions. PloS One. 2014;9(3):e90633.

4. Sharif B, Kopec J, Bansback N, Rahman MM, Flanagan WM, Wong H, Fines P, Anis A. Projecting the direct cost burden of osteoarthritis in Canada using a microsimulation model. Osteoarthr Cartil. 2015;23(10):1654-63.

5. Martin BI, Turner JA, Mirza SK, Lee MJ, Comstock BA, Deyo RA. Trends in health care expenditures, utilization, and health status among US adults with spine problems, 1997-2006. Spine. 2009;34(19):2077-84.

6. Gupta S, Hawker GA, Laporte A, Croxford R, Coyte PC. The economic burden of disabling hip and knee osteoarthritis $(\mathrm{OA})$ from the perspective of individuals living with this condition. Rheumatology. 2005:44(12):1531-7.

7. Maetzel A, Li LC, Pencharz J, Tomlinson G, Bombardier C. The economic burden associated with osteoarthritis, rheumatoid arthritis, and hypertension: a comparative study. Ann Rheum Dis. 2004;63(4):395-401.

8. Xie F, Kovic B, Jin X, He X, Wang M, Silvestre C. Economic and humanistic burden of osteoarthritis: a systematic review of large sample studies. Pharmacoeconomics. 2016:34(11):1087-100.

9. Brosseau L, Taki J, Desjardins B, Thevenot O, Fransen M, Wells GA, Imoto AM, Toupin-April K, Westby M, Gallardo IC, Gifford W. The Ottawa panel clinical practice guidelines for the management of knee osteoarthritis. Part one: introduction, and mind-body exercise programs. Clin Rehabil. 2017; 31(5):582-95.

10. Brosseau L, Taki J, Desjardins B, Thevenot O, Fransen M, Wells GA, Mizusaki Imoto A, Toupin-April K, Westby M, Alvarez Gallardo IC, Gifford W. The Ottawa panel clinical practice guidelines for the management of knee osteoarthritis. Part three: aerobic exercise programs. Clin Rehabil. 2017;31(5): 612-24.

11. Brosseau L, Taki J, Desjardins B, Thevenot O, Fransen M, Wells GA, Mizusaki Imoto A, Toupin-April K, Westby M, Alvarez Gallardo IC, Gifford W. The Ottawa panel clinical practice guidelines for the management of knee osteoarthritis. Part two: strengthening exercise programs. Clin Rehabil. 2017; 31(5):596-611

12. McAlindon TE, Bannuru R, Sullivan MC, Arden NK, Berenbaum F, BiermaZeinstra SM, Hawker GA, Henrotin Y, Hunter DJ, Kawaguchi H, Kwoh K. OARSI guidelines for the non-surgical management of knee osteoarthritis. Osteoarthr Cartil. 2014;22(3):363-88.

13. Fransen M, McConnell S, Harmer AR, Van der Esch M, Simic M, Bennell KL. Exercise for osteoarthritis of the knee: a Cochrane systematic review. $\mathrm{Br} J$ Sports Med. 2015;49(24):1554-7.

14. Bartholdy C, Juhl C, Christensen R, Lund H, Zhang W, Henriksen M. The role of muscle strengthening in exercise therapy for knee osteoarthritis: a systematic review and meta-regression analysis of randomized trials. Semin Arthritis Rheum. 2017;47(1):9-21.

15. Bannuru RR, Osani MC, Vaysbrot EE, Arden NK, Bennell K, Bierma-Zeinstra SM, Kraus VB, Lohmander LS, Abbott JH, Bhandari M, Blanco FJ. OARSI guidelines for the non-surgical management of knee, hip, and polyarticular osteoarthritis. Osteoarthr Cartil. 2019;27(11):1578-89.
16. Crandall S, Howlett S, Keysor JJ. Exercise adherence interventions for adults with chronic musculoskeletal pain. Phys Ther. 2013;93(1):17-21.

17. Marks R. Knee osteoarthritis and exercise adherence: a review. Curr Aging Sci. 2012:5(1):72-83.

18. Voelker R. Few adults with knee osteoarthritis meet national guidelines for physical activity. JAMA. 2011;306(13):1428-30.

19. Marks R, Allegrante JP. Chronic osteoarthritis and adherence to exercise: a review of the literature. J Aging Phys Activ. 2005;13(4):434-60.

20. Holden MA, Nicholls EE, Young J, Hay EM, Foster NE. Role of exercise for knee pain: what do older adults in the community think? Arthritis Care Res. 2012;64(10):1554-64.

21. Stone RC, Baker J. Painful choices: a qualitative exploration of facilitators and barriers to active lifestyles among adults with osteoarthritis. J Applied Gerontol. 2017;36(9):1091-116.

22. Rosemann T, Kuehlein T, Laux G, Szecsenyi J. Factors associated with physical activity of patients with osteoarthritis of the lower limb. J Eval Clin Pract. 2008;14(2):288-93.

23. Srikandarajah S, Gilron I. Systematic review of movement-evoked pain versus pain at rest in postsurgical clinical trials and meta-analyses: a fundamental distinction requiring standardized measurement. Pain. 2011; 152(8):1734-9.

24. Lima LV, Abner TS, Sluka KA. Does exercise increase or decrease pain? Central mechanisms underlying these two phenomena. J Physiol. 2017; 595(13):4141-50

25. Nijs J, Kosek E, Van Oosterwijck J, Meeus M. Dysfunctional endogenous analgesia during exercise in patients with chronic pain: to exercise or not to exercise? Pain Physician. 2012;15(3S):ES205-13.

26. Van Oosterwijck J, Nijs J, Meeus M, Van Loo M, Paul L. Lack of endogenous pain inhibition during exercise in people with chronic whiplash associated disorders: an experimental study. J Pain. 2012;13(3):242-54.

27. Tverskoy M, Oren M, Dashkovsky I, Kissin I. Alfentanil dose-response relationships for relief of postoperative pain. Anesth Analg. 1996;83(2):38793.

28. Mankovsky-Arnold T, Wideman TH, Larivière C, Sullivan MJ. TENS attenuates repetition-induced summation of activity-related pain following experimentally induced muscle soreness. J Pain. 2013;14(11):1416-24.

29. Meeus M, Hermans L, Ickmans K, Struyf F, Van Cauwenbergh D, Bronckaerts L, De Clerck LS, Moorken G, Hans G, Grosemans S, Nijs J. Endogenous pain modulation in response to exercise in patients with rheumatoid arthritis, patients with chronic fatigue syndrome and comorbid fibromyalgia, and healthy controls: a double-blind randomized controlled trial. Pain Pract. 2015;15(2):98-106.

30. Hegedüs B, Viharos L, Gervain M, Gálfi M. The effect of low-level laser in knee osteoarthritis: a double-blind, randomized, placebo-controlled trial. Photomed Laser Surg. 2009;27(4):577-84.

31. Al Rashoud AS, Abboud RJ, Wang W, Wigderowitz C. Efficacy of low-level laser therapy applied at acupuncture points in knee osteoarthritis: a randomised double-blind comparative trial. Physiotherapy. 2014;100(3):242-8.

32. Gur A, Cosut A, Jale Sarac A, Cevik R, Nas K, Uyar A. Efficacy of different therapy regimes of low-power laser in painful osteoarthritis of the knee: a double-blind and randomized-controlled trial. Lasers Surg Med. 2003;33(5):330-8.

33. Cotler HB, Chow RT, Hamblin MR, Carroll J. The use of low level laser therapy (LLLT) for musculoskeletal pain. MOJ Orthop Rheumatol. 2015;2(5): 188-94.

34. Alfredo PP, Bjordal JM, Junior WS, Lopes-Martins RÁ, Stausholm MB, Casarotto RA, Marques AP, Joensen J. Long-term results of a randomized, controlled, double-blind study of low-level laser therapy before exercises in knee osteoarthritis: laser and exercises in knee osteoarthritis. Clin Rehabil. 2018:32(2):173-8.

35. Chow RT, David MA, Armati PJ. $830 \mathrm{~nm}$ laser irradiation induces varicosity formation, reduces mitochondrial membrane potential and blocks fast axonal flow in small and medium diameter rat dorsal root ganglion neurons: implications for the analgesic effects of $830 \mathrm{~nm}$ laser. J Peripher Nerv Syst. 2007:12(1):28-39.

36. Akbari A, Naroii SH, Eshgi M, Farahani A. A comparison between muscle energy technique with low-level laser in reducing neck and shoulder pain and disability in subjects with trapezius and levator scapula myofascial trigger points. J Adv Med Biomed Res. 2012;20(79):69-82.

37. Glazov G, Yelland M, Emery J. Low-level laser therapy for chronic nonspecific low back pain: a meta-analysis of randomised controlled trials. Acupunct Med. 2016;34(5):328-41. 
38. Huang Z, Chen J, Ma J, Shen B, Pei F, Kraus VB. Effectiveness of low-level laser therapy in patients with knee osteoarthritis: a systematic review and meta-analysis. Osteoarthr Cartil. 2015;23(9):1437-44.

39. Huang Z, Ma J, Chen J, Shen B, Pei F, Kraus VB. The effectiveness of lowlevel laser therapy for nonspecific chronic low back pain: a systematic review and meta-analysis. Arthritis Res Ther. 2015;17(1):360

40. Fulop AM, Dhimmer S, Deluca JR, Johanson DD, Lenz RV, Patel KB, Douris PC, Enwemeka CS. A meta-analysis of the efficacy of laser phototherapy on pain relief. Clin J Pain. 2010;26(8):729-36.

41. Whitehead AL, Julious SA, Cooper CL, Campbell MJ. Estimating the sample size for a pilot randomised trial to minimise the overall trial sample size for the external pilot and main trial for a continuous outcome variable. Stat Methods Med Res. 2016;25(3):1057-73.

42. Loew L, Brosseau L, Wells GA, Tugwell P, Kenny GP, Reid R, Maetzel A, Huijbregts M, McCullough C, De Angelis G, Coyle D. Ottawa panel evidencebased clinical practice guidelines for aerobic walking programs in the management of osteoarthritis. Arch Phys Med Rehabil. 2012;93(7):1269-85.

43. Tremblay MS, Warburton DE, Janssen I, Paterson DH, Latimer AE, Rhodes RE, Kho ME, Hicks A, LeBlanc AG, Zehr L, Murumets K. New Canadian physical activity guidelines. App Physiol Nutr Me. 2011;36(1):36-46.

44. Warburton DE, Nicol CW, Bredin SS. Health benefits of physical activity: the evidence. CMAJ. 2006;174(6):801-9.

45. Booth J, Moseley GL, Schiltenwolf M, Cashin A, Davies M, Hübscher M. Exercise for chronic musculoskeletal pain: a biopsychosocial approach. Musculoskeletal Care. 2017;15(4):413-21.

46. Schulz KF, Grimes DA. Sample size slippages in randomised trials: exclusions and the lost and wayward. Lancet. 2002;359(9308):781-5.

47. Thabane L, Ma J, Chu R, Cheng J, Ismaila A, Rios LP, Robson R, Thabane M, Giangregorio L, Goldsmith CH. A tutorial on pilot studies: the what, why and how. BMC Med Res Methodol. 2010;10(1):1.

48. Huntley AL, Johnson R, Purdy S, Valderas JM, Salisbury C. Measures of multimorbidity and morbidity burden for use in primary care and community settings: a systematic review and guide. Ann Fam Med. 2012; 10(2):134-41.

49. Perkins AJ, Kroenke K, Unützer J, Katon W, Williams JW, Hope C, Callahan CM. Common comorbidity scales were similar in their ability to predict health care costs and mortality. J Clin Epidemiol. 2004;57(10):1040-8.

50. Wideman TH, Finan PH, Edwards RR, Quartana PJ, Buenaver LF Haythornthwaite JA, Smith MT. Increased sensitivity to physical activity among individuals with knee osteoarthritis: relation to pain outcomes, psychological factors, and responses to quantitative sensory testing. Pain. 2014;155(4):703-11.

51. Lambin DI, Thibault P, Simmonds M, Lariviere C, Sullivan MJ. Repetitioninduced activity-related summation of pain in patients with fibromyalgia. Pain. 2011;152(6):1424-30.

52. Sullivan MJ, Thibault $P$, Andrikonyte J, Butler $H$, Catchlove $R$, Larivière $C$. Psychological influences on repetition-induced summation of activityrelated pain in patients with chronic low back pain. Pain. 2009;141(1-2):708

53. Wideman TH, Edwards RR, Finan PH, Haythornthwaite JA, Smith MT. Comparing the predictive value of task performance and task-specific sensitivity during physical function testing among people with knee osteoarthritis. J Orthop Sports Phys Ther. 2016;46(5):346-56.

54. Mankovsky-Arnold T, Wideman TH, Larivière C, Sullivan MJ. Measures of spontaneous and movement-evoked pain are associated with disability in patients with whiplash injuries. J Pain. 2014;15(9):967-75.

55. Tan G, Jensen MP, Thornby Jl, Shanti BF. Validation of the brief pain inventory for chronic nonmalignant pain. J Pain. 2004;5(2):133-7.

56. Gandek B. Measurement properties of the Western Ontario and McMaster Universities Osteoarthritis Index: a systematic review. Arthritis Care Res. 2015; 67(2):216-29.

57. Kamper SJ, Maher CG, Mackay G. Global rating of change scales: a review of strengths and weaknesses and considerations for design. J Man Manip Ther. 2009;17(3):163-70

58. Fritz JM, Hebert J, Koppenhaver S, Parent E. Beyond minimally important change: defining a successful outcome of physical therapy for patients with low back pain. Spine. 2009;34(25):2803-9.

59. loannidis JP, Evans SJ, Gøtzsche PC, O'neill RT, Altman DG, Schulz K, Moher D. Better reporting of harms in randomized trials: an extension of the CONSORT statement. Ann Intern Med. 2004;141(10):781-8.
60. Eldridge SM, Chan CL, Campbell MJ, Bond CM, Hopewell S, Thabane L, Lancaster GA. CONSORT 2010 statement: extension to randomised pilot and feasibility trials. BMJ. 2016;355:15239.

61. Naylor JM, Hayen A, Davidson E, Hackett D, Harris IA, Kamalasena G, Mittal R. Minimal detectable change for mobility and patient-reported tools in people with osteoarthritis awaiting arthroplasty. BMC Musculoskelet Disord. 2014;15(1):235.

62. Stratford PW, Kennedy DM, Woodhouse LJ. Performance measures provide assessments of pain and function in people with advanced osteoarthritis of the hip or knee. Phys Ther. 2006;86(11):1489-96.

63. Arendt-Nielsen L, Nie H, Laursen MB, Laursen BS, Madeleine P, Simonsen $\mathrm{OH}$, Graven-Nielsen T. Sensitization in patients with painful knee osteoarthritis. Pain. 2010;149(3):573-81.

64. Fingleton C, Smart K, Moloney N, Fullen BM, Doody C. Pain sensitization in people with knee osteoarthritis: a systematic review and meta-analysis. Osteoarthr Cartil. 2015;23(7):1043-56.

65. Swain S, Sarmanova A, Coupland C, Doherty M, Zhang W. Comorbidities in osteoarthritis: a systematic review and meta-analysis of observational studies. Arthritis Care Res. 2019;72(7):991-1000.

66. Paskins Z, Sanders T, Croft PR, Hassell AB. The identity crisis of osteoarthritis in general practice: a qualitative study using video-stimulated recall. Ann Fam Med. 2015:13(6):537-44.

67. van Dijk GM, Veenhof C, Schellevis F, Hulsmans H, Bakker JP, Arwert H, Dekker JH, Lankhorst GJ, Dekker J. Comorbidity, limitations in activities and pain in patients with osteoarthritis of the hip or knee. BMC Musculoskelet Dis. 2008;9(1):95.

68. Suri P, Morgenroth DC, Kwoh CK, Bean JF, Kalichman L, Hunter DJ. Low back pain and other musculoskeletal pain comorbidities in individuals with symptomatic osteoarthritis of the knee: data from the osteoarthritis initiative. Arthritis Care Res. 2010;62(12):1715-23.

69. Sherman AM. Social relations and depressive symptoms in older adults with knee osteoarthritis. Soc Sci Med. 2003;56(2):247-57.

70. Kendzerska T, Jüni P, King LK, Croxford R, Stanaitis I, Hawker GA. The longitudinal relationship between hand, hip and knee osteoarthritis and cardiovascular events: a population-based cohort study. Osteoarthritis Cartil. 2017;25(11):1771-80.

\section{Publisher's Note}

Springer Nature remains neutral with regard to jurisdictional claims in published maps and institutional affiliations.
Ready to submit your research? Choose BMC and benefit from:

- fast, convenient online submission

- thorough peer review by experienced researchers in your field

- rapid publication on acceptance

- support for research data, including large and complex data types

- gold Open Access which fosters wider collaboration and increased citations

- maximum visibility for your research: over $100 \mathrm{M}$ website views per year

At $\mathrm{BMC}$, research is always in progress.

Learn more biomedcentral.com/submission 